\title{
L'émeute révolutionnaire au travers de manuels de la Troisième République
}

Revolutionary riots through the third republic textbooks

El motín revolucionario a través de manuales de la tercera república

Marc Deleplace

\section{OpenEdition}

Journals

Édition électronique

URL : https://journals.openedition.org/mots/10273

DOI : $10.4000 /$ mots. 10273

ISSN : 1960-6001

Éditeur

ENS Éditions

Édition imprimée

Date de publication : 1 juillet 2002

Pagination : 47-64

ISBN : 2-84788-011-9

ISSN : 0243-6450

\section{Référence électronique}

Marc Deleplace, «L'émeute révolutionnaire au travers de manuels de la Troisième République », Mots.

Les langages du politique [En ligne], 69 | 2002, mis en ligne le 13 mai 2008, consulté le 23 avril 2022.

URL : http://journals.openedition.org/mots/10273 ; DOl : https://doi.org/10.4000/mots.10273

\section{(c) ENS Éditions}




\section{L'émeute révolutionnaire au travers de manuels de la Troisième République}

L'émeute révolutionnaire ${ }^{1}$ occupe, dans les manuels de la Troisième République, une place à la fois centrale et ambigüe. Centrale parce qu'on lui reconnait, que ce soit pour communier dans la célébration ou pour s'en désoler, la valeur d'un acte fondateur du nouvel ordre des choses. Mais ambigüe parce qu'en tant qu'émeute, elle véhicule, pour ceux-là mêmes qui se revendiquent d'une partie au moins de l'héritage révolutionnaire, l'image d'un désordre inquiétant que l'on n'est pas sûr de pouvoir légitimer dans l'espace politique autrement que par son résultat. Cette situation se traduit, au-delà d'importantes divergences liées à l'acceptation ou non du fait révolutionnaire comme fondement de la nation républicaine, voire de la reconnaissance ou non de la légitimité républicaine, par le partage d'une défiance pouvant se muer en désapprobation plus ou moins fortement exprimée à l'encontre de l'émeute révolutionnaire.

De ce point de vue, le récit des évènements de 1789 et 1792 peut aussi bien relever d'un récit historiographique à caractère didactique, fonction première du manuel scolaire, que de l'expression détournée d'une interrogation sur la légitimité et la légitimation révolutionnaire de l'émeute. C'est ce deuxième aspect qui nous retiendra ici, étudié par la confrontation entre deux manuels contemporains, mais représentant deux positions antinomiques autour de la Révolution : le manuel classique de Jules Isaac publié sous le titre de Nouveau cours d'Histoire Malet-Isaac, en $1928^{2}$; le manuel de Charles Aimond, publié par l'éditeur catholique Jean de Gigord en $1925^{3}$.

\footnotetext{
- IUFM de Reims, 23 rue Clément Ader, 51100 Reims

1. Nous utilisons cette expression comme terme générique non référencé dans le corpus.

2. Il succède, au Cours d'histoire publié en 1908 par A. Malet à la demande d'E. Lavisse et dont J. Isaac était déjà l'un des auteurs.

3. Le fait que le second soit publié en référence au programme de 1902 alors que le premier répond au nouveau programme de 1923 ne change rien à la place qu'y occupe la Révolution
} 
Si la position de ce dernier auteur, résolument hostile à la Révolution, invalide immédiatement l'émeute révolutionnaire, de sorte qu'il est difficile de parler de débat, cette confrontation permet de saisir comment, chez un auteur favorable à la Révolution comme l'est Isaac, l'acceptation de l'émeute peut être rendue difficile. C'est-à-dire pour saisir comment le récit des évènements révolutionnaires peut jouer comme représentation sociale ${ }^{4}$, et l'émeute révolutionnaire comme représentation négative.

Le récit des journées révolutionnaires peut être qualifié de « représentation sociale » à partir du moment où il véhicule des conceptions générales sur l'ordre politique et social qui dépassent le cadre historiographique assigné ${ }^{5}$. Ce glissement du récit descriptif au débat sociopolitique se fait autour de deux interrogations implicites qui posent le problème de la légitimité de l'émeute révolutionnaire, interrogations déjà présentes dans les débats révolutionnaires eux-mêmes. La première, l'émeute comme acte de souveraineté, se lit au travers de la mise en scène des acteurs, des formes de manifestations adoptées et des buts exposés. La seconde, celle de la violence populaire, fragilise incontestablement la position de l'émeute dans le récit historiographique républicain. Elle ressort également de la précédente dans la mesure où elle touche à la question de l'exercice de la souveraineté par le biais du droit à l'insurrection, débat très vif en l'an III alors que la Convention nationale est en but aux ultimes soubresauts du mouvement populaire ${ }^{6}$. Autrement dit, l'émeute révolutionnaire met en jeu la souveraineté et l'ordre social, et son évocation, fut-elle historiographique, mobilise des modèles politiques et sociaux.

française. Ajoutons que ces deux manuels sont comparables quant aux conditions de leur écriture. Les auteurs appartiennent tous au milieu universitaire : J. Guiraud, qui dirige la publication du manuel de Ch. Aimond, professeur agrégé, chez J. de Gigord, est Inspecteur général de l'Instruction publique, position à laquelle accédera par la suite J. Isaac; ce dernier est également professeur agrégé comme son devancier A. Malet, et s'adjoint pour le volume de première Ch.$\mathrm{H}$. Pouthas, docteur ès lettres et futur professeur à la Sorbonne. Ils se distinguent en revanche par les références historiographiques qui fondent leur propos : Aulard, Sagnac et Mathiez pour Malet et Isaac, Sagnac toujours mais surtout H. Taine pour Aimond.

4. Pour ce que nous mettons derrière la notion de "représentations sociales ", nous renvoyons d'une manière générale à l'article fondateur de R. Chartier «Le Monde comme représentation ", Annales ESC, 6, 1989, ainsi qu'à D. Jodelet, 1989, Les Représentations sociales, PUF.

5. Nous nous inspirons ici partiellement de l'article d'A. Bourreau, « La compétence inductive. Un modèle d'analyse des représentations rares », dans B. Lepetit (dir.), 1995, Les Formes de l'expérience. Une autre histoire sociale, Albin Michel.

6. Cette double question de la signification des acteurs et de la violence populaire se rencontre notamment chez M. Vovelle, 1985, La Mentalité révolutionnaire, Messidor-Éditions sociales ; R. Cobb, 1975, La Protestation populaire en France, Calmann-Lévy ; ou G. Rudé, 1982, Les Foules de la Révolution française, Maspero. 
Faisant ainsi fonction de « représentation sociale », l'émeute révolutionnaire joue également le rôle d'une représentation négative. Nous entendons sous cette appellation des représentations qui se construisent différemment de celles qui ont jusqu'à présent retenu l'attention en ce qu'elles ne donnent pas lieu à une élaboration explicite qui permet aux acteurs de les mobiliser en bloc et, par suite, au chercheur de les identifier pour ce qu'elles sont, ce qui ne les empêche nullement de jouer un rôle important dans la structuration du discours sociopolitique. Du moins est-ce l'hypothèse formulée en conclusion de notre travail sur la notion d'anarchie, en cherchant à prolonger l'idée d'une anarchie érigée en anti-modèle social en l'an III. Ces représentations négatives se caractériseraient donc par le fait qu'elles apparaissent sous la forme d'énoncés éclatés, qu'elles sont également diffuses et largement partagées, donc moins nettement liées à un groupe ou une catégorie sociale ou sociopolitique déterminée, ce qui en dernière instance les rendrait à la fois résistantes et instables dans le champ du discours politique ${ }^{7}$. Elles apparaissent donc essentiellement comme des figures en creux, telle l'anarchie dans le discours politique révolutionnaire, ce qui ne les renvoie pas pour autant à l'indétermination pure et simple ${ }^{8}$.

Trois éléments sont décisifs pour mesurer la position de l'émeute révolutionnaire dans le discours des manuels : la désignation et la mise en scène des acteurs ; l'ordre du récit ; les relations de causalité et l'arrièreplan interprétatif qu'elles suggèrent ${ }^{9}$.

7. 1. Résistantes : précisément parce que suffisamment partagées pour rendre difficile les processus de retournement. L'exemple de Proudhon, affronté à ce problème, dans sa volonté de redéfinir positivement l'anarchie dans le discours politique, le montre bien. Il ne peut faire l'économie d'une configuration négative de l'anarchie, élaborée pour l'essentiel en l'an III, au point de la reprendre parfois à son compte. Ce qui introduit un flottement de ses énoncés en termes d'anarchie, perçu par certains de ses critiques comme une palinodie de sa pensée. 2. Instables : dans la mesure où de telles configurations négatives peuvent être mobilisées de manière contradictoire dans certains débats, telle l'anarchie renvoyée d'un banc à l'autre des assemblées révolutionnaires, pour invalider successivement ou simultanément la république et la monarchie. Voir sur ces deux aspects : M. Deleplace, 2001, L'Anarchie de Mably à Proudhon (1750-1850), Lyon, ENS-Éditions, (notamment les chapitres 5 et 7 pour le premier aspect, le chapitre 3 pour le second, et la conclusion pour une première approche de la notion de représentation négative).

8. Contrairement au jugement formulé sur la notion d'anarchie pendant la Révolution par F. Brunot, 1937, Histoire de la langue Française, A. Colin, tome 9, p. 827 ; et repris par J. Maitron, 1983, (1975), Le Mouvement anarchiste en France, Maspéro, p. 14. Voir M. Deleplace, 2001, op. cit., p.11-13, Lyon, ENS-Éditions, Introduction, p. 11-13.

9. Nous nous sommes concentrés sur les récits des journées de 1789 (14 juillet, 5 et 6 octobre) et de 1792 (10 aout et massacres de septembre). Les passages étudiés sont les suivants :

- 14 juillet : Isaac, p. 65-68; Aimond, p. 274-276 ;

- 5 et 6 octobre : Isaac, p. 72-74; Aimond, p. 279-281;

- 10 aout et massacres de septembre : Isaac, p. 124-132 ; Aimond, p. 312-317. 


\section{La désignation et la mise en scène des acteurs}

Chacun des auteurs se livre à une énumération qui révèle l'acceptation ou le rejet de la légitimité de l'émeute révolutionnaire. Cela se marque à plusieurs niveaux : la désignation des acteurs ; le registre du vocabulaire de description des groupes ; la mobilisation du collectif « peuple ».

Les auteurs partagent une égale retenue à l'égard des individus. Les notations qui les concernent sont peu fréquentes et moins appuyées que pour les acteurs collectifs. Tout au plus peut-on relever chez Isaac une appréciation concernant « le populaire Danton», au 10 aout 1792. Le plus souvent, c'est en relation avec une mention visant un groupe d'acteurs que l'on peut percevoir l'expression d'une préférence. Ainsi de la présentation de Camille Desmoulins, le 12 juillet 1789. Pour Isaac, la figure du « jeune écrivain » se détache d'un groupe « d'orateurs improvisés » haranguant la foule du Palais-Royal, tandis que pour Aimond le « jeune tribun » est le plus en vue des « orateurs populaires » qui « surexcitaient cette foule impressionnable ».

Cette subordination de l'individu au collectif est sans doute l'effet des impératifs de concision d'un manuel scolaire. Aussi est-ce la galerie des acteurs collectifs qui donne le ton. La nuance introduite dans la présentation des orateurs du Palais-Royal par les deux adjectifs « improvisés » et «populaires » marque l'écart qui sépare de légitimes porte-parole ${ }^{10}$ du peuple souverain des démagogues qui cherchent à l'égarer en jouant de ses passions.

La prédilection des auteurs pour un certain registre de qualification des groupes d'acteurs marque plus nettement ce qui les sépare. Aimond se cantonne dans le domaine social, ignorant le registre politique qu'Isaac introduit au contraire doublement : dans le recours à une désignation sociopolitique plus positive, et dans la mention régulière des autorités constituées.

Cette combinaison se retrouve au 14 juillet comme au 10 aout, ce qui donne une place centrale à la formation d'une commune insurrectionnelle. Dans le premier cas, deux organes sont le prétexte à la présentation des acteurs : la municipalité formée le 13 juillet et la milice bourgeoise, préfiguration de la Garde nationale. De la première, Isaac

10. La figure du porte-parole est décisive dans la légitimation révolutionnaire du mouvement populaire : voir J. Guilhaumou, 1998, L'Avènement des porte-parole de la République (17891792), Lille, Presses universitaires du Septentrion. C'est en partie faute de pouvoir concevoir cette figure, malgré cette fugitive notation, que J. Isaac peine à légitimer l'émeute révolutionnaire. 
explique qu'elle provient d'un comité permanent constitué du «Bureau de Ville» (Prévôt des marchands et échevins) et des « Électeurs du Tiers » (la majuscule donnant valeur de groupe constitué). Quant à la milice, elle comptait les "meilleurs citoyens de la ville »: "nobles, bourgeois, financiers, prêtres même ». Une énumération qui montre la légitimité de l'acte insurrectionnel par la capacité politique et sociale avérée des acteurs, et par leur caractère représentatif de l'ensemble du corps politique.

C'est le sens que l'on peut également donner à la présentation des forces de l'émeute du 10 aout, bien qu'elle s'exprime dans un lexique sensiblement différent : ce sont ici les Jacobins et les Cordeliers, puis les sociétés populaires et les assemblées des sections de Paris, enfin les fédérés des provinces, qui sont nommés. Mais comme au 13 juillet, c'est la formation d'une commune par délégation représentative qui clôt cette énumération et légitime les acteurs de l'insurrection.

Des commissaires, élus par les sections, s'installaient à l'Hôtel de Ville [...]. Une fois dans la place, ils formèrent une sorte de commune insurrectionnelle qui prit la direction du mouvement ${ }^{11}$.

Rien de tel chez Aimond, bien qu'il reprenne les mêmes éléments : les fédérés et les sections parisiennes (mais pas les «assemblées » de ces sections, ni les clubs, ni les sociétés populaires, autrement dit, il ne reconnait aucune valeur politique aux organisations du mouvement populaire). Il insiste sur la formation de la commune mais pour lui donner une tout autre connotation.

Le tocsin commença à soulever les faubourgs et des bandes armées vinrent se grouper devant l'Hôtel de Ville. Celui-ci fut envahi par les commissaires des sections parisiennes, qui en expulsèrent les officiers municipaux et constituèrent de leur propre autorité, la célèbre Commune insurrectionnelle.

Le registre de vocabulaire employé interdit de leur accorder une quelconque légitimité. Il n'y a plus ici qu'usurpation d'autorité.

L'écart est plus grand encore dans la description des émeutiers du 14 juillet 1789. Ceux-ci n'apparaissent que sous le qualificatif de « bandes d'insurgés ». La seule notation qui en précise le visage est faite indirectement lorsqu'Aimond relève, dans les jours qui précèdent, « l'afflux incessant de vagabonds et d'ouvriers sans travail ». La disqualification des acteurs de l'émeute ainsi opérée est redoublée par leur opposition à la seule force insurrectionnelle organisée dont Aimond fait mention, la milice bourgeoise, formée par les « Parisiens effrayés » des désordres de leur ville.

11. Dans les citations, les passages en italique sont soulignés par les auteurs des manuels. 
Le dernier aspect de cette question réside dans la mobilisation ou non de la figure du peuple souverain. L'enjeu est de poser les conditions auxquelles l'émeute révolutionnaire peut être qualifiée d'acte de souveraineté, seule façon de la légitimer dans le champ politique. D'une part, cela suppose l'identification des acteurs au peuple souverain tout entier, dans une recherche d'unanimité, fondée sur l'indivisibilité, que l'on rencontre dans la Révolution elle-même. D'autre part, il faut qu'il existe, au sein même de l'émeute, une possibilité de médiation institutionnelle.

Aimond nous présente un tableau sans nuances sur ces deux points. Nulle part il ne voit l'action d'un peuple souverain : ce " peuple » qui n'en est pas un s'oppose aux autorités constituées, y compris celles susceptibles d'assurer la médiation de ses demandes. Le 14 juillet 1789, ce n'est pas «le Peuple », mais «plus spécialement le bas-peuple » qui entre en scène. Camille Desmoulins excite « la populace à la révolte et au pillage ». Aimond poursuit sur sa lancée en octobre : ce n'est en rien le peuple souverain mais « une foule de 6000 à 7000 femmes des Halles, trainant des canons et mêlée d'individus sans aveu » qui se rend à Versailles. Et le 10 aout ne corrige en rien cette triste réalité : «l'armée de l'émeute devait se recruter, comme à l'ordinaire, dans les faubourgs ", même s'il concède qu'elle « devait être renforcée par les fédérés ». L'absence d'unanimité est redoublée de l'opposition continue aux formes institutionnalisées, toujours placées en position défensive. En juillet 1789, " les Parisiens effrayés organisaient une milice bourgeoise ». En aout 1792, « les Girondins, effrayés de ces préparatifs, essayèrent, mais en vain, de se rapprocher de la Cour $»$.

La position d'Isaac est plus complexe et rend compte de la difficulté à légitimer l'émeute. Chez ce dernier, l'énumération des forces de l'émeute vise à convaincre de leur aptitude à représenter le peuple souverain en action, caractère nécessaire à sa légitimation. S'il use avec parcimonie du collectif " peuple », du moins le place-t-il en des endroits stratégiques de son récit. La présentation des journées de juillet s'ouvre par une mention du « peuple de Paris » qu'il célèbre par la suite comme le vainqueur de la Bastille. Un évènement auquel son retentissement confère en lui-même valeur d'acte de souveraineté.

La Bastille, prison d'État, était le symbole de l'arbitraire. La prise de la Bastille par le peuple de Paris eut un immense retentissement. Elle fut l'évènement décisif qui entraina l'effondrement du pouvoir absolu et de tout l'Ancien Régime, politique et social.

L'expression est plus ample encore au 10 aout, après qu'Isaac a rappelé les dangers courus par suite de l'invasion étrangère. 
De pareilles menaces ne pouvaient que soulever Paris et la France, dans un élan de colère patriotique, contre le roi, évidemment complice de l'étranger.

L'union de Paris et de la France, manifestée par la participation des sections et des fédérés à la prise des Tuileries, garantit semble-t-il sans hésitation la validité de l'acte accompli. Et pourtant, déjà sur ce point perce l'incertitude.

En effet, la seconde condition de validation de l'émeute, la possibilité d'une médiation par une autorité constituée, n'est plus remplie le 10 aout. Isaac met au contraire en avant, face à l'inertie de l'Assemblée, un ensemble d'acteurs représentatifs d'un peuple souverain qui se mobilise pour pallier l'insuffisance de ses représentants, les dessaisissant du même coup.

Ce fut hors de l'Assemblée et malgré elle que les révolutionnaires les plus décidés, Jacobins et Cordeliers, préparèrent l'insurrection qui devait jeter bas la royauté. Ils eurent pour eux, avec les sociétés populaires et les assemblées des sections de Paris, les fédérés appelés de province, malgré le veto du roi, pour l'anniversaire de la fête de la Fédération.

Nous disposons ainsi de deux critères, unanimité du corps souverain et union avec les représentants légitimes, qui permettent d'établir un classement de recevabilité des émeutes révolutionnaires chez Isaac. Classement dont le premier terme est occupé par le 14 juillet 1789 qui réunit harmonieusement ces deux critères, tandis que les journées d'octobre en occupent le dernier.

Du premier, Isaac écrit que «Le peuple de Paris sauva l'Assemblée menacée, et son intervention assura le triomphe de la Révolution ». Quant aux secondes, si Isaac conserve à la foule qui se dirige sur Versailles un caractère de plus grande dignité qu'Aimond, il ne masque pas la double rupture de l'unanimité : la Garde nationale vient à part de la foule (la formation de cette garde est dans le récit du 14 juillet l'un des éléments clés de la manifestation de l'unanimité souveraine) et s'oppose le 6 octobre aux émeutiers (comme pour Aimond dès le 14 juillet) ; la foule qui se porte vers l'Assemblée est loin de s'y livrer à une démonstration d'union (« l'Assemblée fut envahie ») ${ }^{12}$.

12. La conscience d'une rupture par rapport au 14 juillet est vive alors que le peuple pour la première fois interrompt les travaux de l'Assemblée, selon T. Tackett, 1997, Par la volonté du peuple, Albin Michel (Princeton University Press, 1996), p. 187 et suiv. On pourrait ajouter que le caractère politique de la journée tend à s'effacer quelque peu y compris par la réduction du sens du slogan "du pain" chez Aimond, slogan qu'Isaac ne reprend du reste pas. Sur le sens politique des énoncés produits autour du pain, voir J. Guilhaumou, D. Maldidier, R. Robin, 1994, Discours et archive, Liège, Mardaga, p. 133 et suiv. Or les registres de désignation des acteurs montre par ailleurs, nous l'avons vu, que la désignation sociale prend une valeur négative. Effacer le politique derrière le social est une manière de dénégation de légitimité. 


\section{L'ordre du récit}

Un constat préliminaire s'impose : le manuel scolaire répond à un genre dont les règles sont suffisamment établies pour que les auteurs y souscrivent également. Ce qui se traduit par un ordre « canonique » de présentation des faits, puisque c'est de cela qu'il s'agit dans une optique définie notamment par Lavisse et inspirée de l'historiographie « positiviste ». Cependant, les découpages adoptés, marqués par les titres et sous-titres, connaissent des variantes dans lesquelles s'inscrivent discrètement les positions des auteurs.

Ainsi pour le récit du 14 juillet, Isaac distingue successivement: « Préparatifs d'un coup d'État», qui met l'accent sur la responsabilité du roi et de son entourage ; «Journées de juillet», centré en fait sur la formation de la municipalité et de la milice bourgeoise ; "Prise de la Bastille »; «Les conséquences ». Aimond ne retient que deux moments : «La prise de la Bastille (14 juillet)» qui fait la part belle à l'émeute et minore les circonstances, et «Les conséquences du 14 juillet ».

Pour le 10 aout, le découpage des parties introduit deux points de vue interprétatifs. Aimond le renferme dans trois paragraphes : «La préparation du 10 aout »; "La journée du 10 aout et ses conséquences »; « Massacres de septembre, fin de la Législative ». Isaac est plus prolixe. Notamment, il intercale deux paragraphes: l'un avant le récit du 10 aout, intitulé « Le manifeste de Brunswick », qui insiste sur les justifications de l'émeute et les fautes du roi ; l'autre après, comme « tampon » entre le 10 aout et les Massacres de septembre qui sont ainsi dissociés, intitulé «La dictature de la commune ». Enfin, l'articulation générale des deux chapitres incluant le 10 aout et intitulés «L'Assemblée législative », renforce cet effet interprétatif. Le chapitre d'Aimond se conclut sur l'évocation des Massacres de septembre, des mesures dirigées contre le clergé et les congrégations religieuses, et in extremis de Valmy (" [L'Assemblée législative] se sépara le 20 septembre 1792, le jour même de la bataille de Valmy »). Isaac développe au contraire la situation extérieure sur trois paragraphes dont deux consacrés à Valmy («Valmy » et « Conséquences de Valmy ») et tait les mesures religieuses.

En second lieu, des variations et des déplacements dans l'ordre du récit peuvent renforcer l'effet de jugement induit par l'économie des paragraphes. Deux exemples permettent de s'en faire une idée. Ils touchent à l'un des critères mis en évidence précédemment, celui du 
rapport aux autorités constituées, et par-delà à l'ordre social, et ouvrent la question de la violence populaire.

Le premier est pris dans le récit du 14 juillet. Les manuels suivent une trame chronologique qui supporte a priori peu de variations : ils déroulent les évènements depuis le 12 jusqu'au 17 juillet, presque jour par jour. En particulier, ils exposent en détail les rassemblements du PalaisRoyal et la charge du Royal-Allemand le 12 et la prise de la Bastille le 14. La pierre d'achoppement est la journée du 13 juillet : pivot du récit chez Isaac ; quasi absente chez Aimond. Ce dernier enchaine les premières échauffourées avec la formation de la milice bourgeoise sans signifier que ces deux évènements ne se produisent pas le même jour.

Une première escarmouche eut lieu près des Champs-Élysées, entre les émeutiers et les dragons royaux qui reculèrent. Devenues maitresses de la ville, les bandes insurgées coururent aux dépôts d'armes, et se mirent à commettre toutes sortes de violences et de pillages, tandis que les Parisiens effrayés organisaient une milice bourgeoise.

D'autre part, il rejette la formation de la nouvelle municipalité dans le paragraphe sur les conséquences du 14 juillet, et sans relation de cause à effet : « À Paris, une nouvelle municipalité s'organisa spontanément et mit le député Bailly à sa tête ».

Tout autre est la position d'Isaac. Il insiste sur l'importance de la journée du 13 juillet, bien distinguée des troubles de la veille : « Le lendemain 13 juillet, il se produisit deux faits d'importance : la formation d'une municipalité révolutionnaire, et la levée d'une milice bourgeoise ». Il introduit par anticipation le terme de garde nationale, dont la milice constitue les premiers éléments. Ce faisant, il donne un tout autre sens à l'organisation de ces deux autorités par rapport à l'émeute. Pour Aimond, la milice se forme par crainte des troubles tandis que la mise en place de nouvelles autorités municipales n'est pas formellement liée à l'émeute. Isaac restitue au contraire le sens politique de l'émeute et lui attribue une capacité d'organisation et de conservation de l'ordre social, nécessaire à son acceptation.

Le traitement réservé à l'épisode de la mort de Mandat, à la veille du 10 aout 1792, est tout autant révélateur. Aimond écrit que :

Le maire, Pétion, déserta son poste, tandis que Mandat, commandant la Garde nationale, était appelé à l'Hôtel de ville et emprisonné, en attendant d'être lâchement massacré.

Aucun de ces actes ne peut trouver de légitimité, ils relèvent également du désordre et de l'arbitraire. À ce récit vibrant d'indignation contenue, Isaac oppose que : 
Le maire Pétion, apeuré et décidé à laisser faire, restait chez lui sous bonne garde. Mandat, convoqué dans la nuit à l'hôtel de ville, eut l'imprudence de s'y rendre : il fut arrêté, destitué, remplacé par Santerre, l'un des meneurs des faubourgs.

C'est par un appel de note au mot destitué qu'Isaac ajoute que « quelques heures plus tard, en sortant de l'Hôtel de ville pour aller en prison, il fut massacré par le peuple ». Les actes sont ainsi nettement séparés : la destitution de Mandat (par une commune insurrectionnelle dont on a vu comment Isaac la légitimait) est un acte légal qui ne contient pas en lui-même le massacre, lequel reçoit du reste un élément de légitimité puisqu'accompli, une fois n'est pas coutume, par «le peuple $»$.

\section{Relations de causalité et interprétation}

Nous avons constaté que les titres des paragraphes s'expriment dans un lexique commun, validé par l'historiographie contemporaine. C'est ainsi qu'ils qualifient tous les deux les évènements décrits de « journées populaires », les Massacres de septembre donnant lieu à une autre dénomination qui en marque la spécificité. Deux remarques doivent être faites à ce propos.

La première est que l'expression n'est pas rigoureusement identique. Aimond seul utilise l'expression adjectivée (Isaac écrit simplement « les journées de juillet »). Si l'on se réfère aux mentions qu'il fait du peuple ( " la populace », " le bas-peuple »), on peut en inférer une connotation péjorative. D'autant que le décalage d'expression entre les deux auteurs se prolonge dans l'évocation du 10 aout : « journée du dix aout » pour Aimond, mais « insurrection du dix aout» pour Isaac.

Tout au long de son récit, Aimond n'emploie, comme équivalent au mot journée, que le terme d'émeute, et désigne les acteurs comme émeutiers, termes chargés négativement si l'on s'en rapporte aux définitions en vigueur depuis la fin du $19^{\mathrm{e}}$ siècle.

Émeute : Mouvement tumultueux dans lequel le peuple, ou une fraction du peuple, et le plus souvent une populace ignorante ou égarée, exprime son mécontentement, soit de la marche, soit de quelque mesure du gouvernement, tantôt par des clameurs menaçantes, tantôt par des actes de violence.

Émeutier : Mot créé depuis 1830 pour ceux qui faisaient partie des émeutes. Ce fut alors qu'on créa un mot nouveau, celui d'émeutier, pour quelques mauvais sujets et certain nombre de gamins de la capitale toujours disposés, les uns à former le noyau du mouvement, les autres à se grouper autour. 
Au contraire, le fait pour Isaac de qualifier l'évènement d'insurrection, est un élément fondamental de valorisation et de reconnaissance dans le champ de l'action politique.

Insurrection : Soulèvement contre le gouvernement. En employant ce mot, on y attache ordinairement une idée de droit et de justice ${ }^{13}$.

Cela étant, deux autres points rendent compte à la fois des positions divergentes des auteurs devant la Révolution française, et du partage d'une même conception de l'ordre social et de sa nécessaire préservation, qui se marque dans la désapprobation indignée d'Aimond et dans les réticences d'Isaac, que l'on peut restituer au travers de certaines énonciations. Il s'agit en premier lieu de l'appréciation du degré de « spontanéité » du « peuple » dans l'émotion, en second lieu de la question de la violence. Nous touchons donc d'une part à la causalité inscrite dans la description de l'évènement, de l'autre au sens général de l'évènement.

Deux types de causes déterminent la ligne de partage entre légitimité et illégitimité de l'émeute révolutionnaire. Soit l'émeute est la réponse directe du peuple aux manifestations de l'oppression, auquel cas, et sous les conditions d'unanimité du peuple et d'union avec ses représentants, l'émeute est acte de souveraineté légitime et célébrée comme libératrice. Soit elle est fomentée par des agitateurs, démagogues de tout poil, elle devient alors irrecevable. Cette ligne de partage est respectée par les deux auteurs si l'on considère le soin que met Isaac à identifier les causes objectives de l'émeute, y compris en octobre 1789 (ce n'est qu'en septembre 1792 qu'il y renonce), et l'insistance d'Aimond à montrer les démagogues à l'œuvre depuis juillet 1789 .

Et pourtant, les causes directes des évènements sont identifiées dans les mêmes faits. En juillet 1789, sont invoqués le renvoi de Necker et les mouvements de troupes; en octobre le conflit entre le roi et l'Assemblée et le banquet des gardes du corps ; en aout 1792, la Patrie en danger et le manifeste de Brunswick. Mais le traitement qui leur est réservé marque nettement les différences. Trois situations peuvent se présenter, qui renvoient toutes peu ou prou à la question des menées subversives d'un groupe d'agitateurs exploitant les peurs populaires.

Il peut y avoir désaccord sur les causes. Ainsi pour le 10 aout, Aimond incrimine au premier chef le décret de l'Assemblée déclarant la Patrie en danger (11 juillet 1792), suscitant « dans tout le pays une émotion intense qu'augmenta encore le cérémonial dramatique adopté pour l'enrô-

13. Bescherelle, Dictionnaire national ou dictionnaire universel de la langue française, Garnier Frères, $1871,14^{\mathrm{C}}$ édition, tome 1. 
lement des volontaires ». L'Assemblée est cause du désordre des esprits par son action intempestive. Le manifeste de Brunswick n'intervient que de manière incidente, alors que pour Isaac : «La réponse au manifeste de Brunswick fut l'insurrection du dix aout».

On peut discuter en second du degré de manipulation de la foule. Les menées ténébreuses des agitateurs supposent à la fois leur séparation d'avec le corps politique et social, et leur assimilation au complot factieux dont le but ne peut être que la subversion de l'ordre social et non sa refondation. Ces meneurs sont déjà présents, pour Aimond, à la veille du 14 juillet, parmi ces « orateurs populaires » qui manipulent la « foule impressionnable » à la faveur des mouvements de troupes qui inquiètent la capitale : « Au Palais-Royal, un jeune tribun, Camille Desmoulins, en prit prétexte pour exciter la populace à la révolte et au pillage ». Mais c'est surtout le 10 aout que « les meneurs, comme Danton, Manuel et Santerre » donnent leur pleine mesure en dérivant « contre la personne de Louis XVI l'indignation causée par le manifeste de Brunswick ». Isaac n'ignore ni les préparatifs ni les cadres de l'insurrection. Mais il n'y voit nullement des menées séditieuses. Il n'établit aucune relation formelle entre les discours des orateurs du Palais-Royal et la prise d'arme des Parisiens. S'il en va un peu différemment pour le 10 aout, puisque l'insurrection est préparée par « les révolutionnaires les plus décidés » et que c'est à leur " instigation » que les fédérés et les sections adressent des pétitions réclamant la déchéance du roi, la commune ne fait que «prendre la direction du mouvement », ce qui suppose son antériorité et son indépendance.

Enfin, Aimond conteste la réalité d'une réaction spontanée à l'oppression quand l'évènement qui détermine l'émeute n'est plus de l'ordre de l'objectif mais de celui du psychologique. En octobre 1789, il ne laisse planer aucun doute quant à l'action d'agitateurs profitant de la rumeur engendrée par le banquet des gardes du corps (« On prétendit que [...] certains royalistes exaltés avaient foulé aux pieds la cocarde tricolore. Cet incident, aussitôt connu à Paris et exagéré par la malveillance, y causa une grande effervescence $»)$. Isaac reconnait certes l'incertitude des récits («on raconta que la cocarde tricolore avait été foulée aux pieds ») mais cette remarque intervient en dehors du cours principal du récit qui insiste au contraire sur le fait que « les convives manifestèrent leur loyalisme monarchique, leur hostilité à l'Assemblée ».

Reste la délicate question de la violence populaire. Elle est présente tout au long des journées révolutionnaires et culmine dans les Massacres de septembre. Trois procédés permettent à Isaac d'en limiter la portée : la séparation des auteurs de ces violences d'avec le «peuple» souve- 
rain ; l'atténuation du caractère criminel des actes accomplis ; le rejet de la violence aux marges de l'évènement.

Le 14 juillet, c'est une "populace en fureur » et non le peuple vainqueur de la Bastille qui massacre le gouverneur de Launay et Flesselles, la référence au prétexte du massacre des deux hommes ("accusés de trahison ») et l'insistance sur la dureté du combat corrigeant la gratuité de l'acte. Et si « le mardi 6 octobre [1789], la situation devint tragique », ce ne sont que « des émeutiers » qui « parvinrent à forcer une des entrées du château [et] tuèrent les gardes du corps qui leur barraient le passage ». Autant de procédés rejetés par Aimond. Il n'existe aucune différence de nature entre les acteurs de l'émeute et les massacreurs. C'est « un millier d'émeutiers » qui occupe la scène depuis l'attaque contre la Bastille jusqu'aux meurtres de Foulon et Berthier, en passant par ceux de Flesselles et de Launay " odieusement égorgés » (évènements traités en un seul paragraphe dont ce collectif est l'unique sujet agissant). Le 6 octobre 1789, " vers 6 heures du matin, les émeutiers s'emparèrent d'une porte du château, massacrèrent les gardes du corps qui veillaient à l'entrée des appartements de la reine ».

Mais le choix interprétatif décisif se lit dans l'inscription de la violence au cœur même de l'émeute révolutionnaire. C'est là que réside le plus grand danger d'invalidation.

Pour Aimond, le meurtre du gouverneur de Launay est inscrit dans la logique de l'évènement, et pour le démontrer, il réduit l'importance de l'engagement. "Attaqué à l'improviste, le gouverneur de Launay fit pour la forme une courte résistance ». Le fait qu'il fut massacré «malgré la parole donnée » place le meurtre comme raison d'être ultime de la journée. L'adjonction des meurtres de Foulon et de Bertier renforçant encore cette certitude.

Isaac met au contraire en scène une lutte déclenchée « dans des conditions mal connues » et beaucoup plus soutenue, puisque ce n'est qu'au terme « de quatre heures d'un combat où les assaillants eurent environ 200 hommes tués ou blessés » que la garnison rend les armes. Surtout, il isole l'épisode du massacre de Flesselles et de Launay :

On ne put empêcher que le triomphe populaire fût souillé par des actes de sauvagerie. Le gouverneur de Launay, le prévôt des marchands Flesselles, accusés de trahison, furent massacrés par une populace en fureur et leurs têtes promenées au bout des piques.

Et passe totalement sous silence les deux meurtres du 22 juillet. La fureur peut s'excuser dans le moment de la violence exercée contre les justes revendications du peuple, elle devient inacceptable dans les jours qui suivent. 
Plus délicate est la question du massacre des prisonniers entre le 2 et le 6 septembre 1792, et de ses relations avec l'insurrection du 10 aout. Pour Aimond, aucune hésitation, le massacre est dans la continuité des actions entreprises par les nouvelles autorités parisiennes, dont il n'admet à aucun moment le caractère légitime ${ }^{14}$. Il laisse supposer un plan concerté :

La Commune avait préparé cette exécution sanglante, en remplissant les prisons de milliers de « suspects », arrêtés au cours des visites domiciliaires. Elle déchaina ensuite les fureurs populaires, en exploitant les graves évènements dont la région de l'Est venait d'être le théâtre [trahison de La Fayette et investissement de la place de Verdun par les Prussiens].

\section{Suit le récit du massacre :}

Le 2 septembre, une troupe d'émeutiers se porta sur les différentes maisons de détention : les Carmes, l'Abbaye, la Conciergerie. Aux Carmes, elle massacra 114 ecclésiastiques (dont 3 évêques) ; à l'Abbaye, 318 prisonniers ; à la Conciergerie, 578. On estime que du 2 au 6 septembre près de 1400 de ces malheureux furent égorgés sans défense, après un simulacre de jugement.

Isaac restitue une tout autre logique aux évènements. Certes :

Une autre conséquence du Dix aout, ce fut la dictature exercée par la Commune de Paris, et, à son inspiration, le développement des mesures d'exception, de ce qu'on peut appeler la politique révolutionnaire de salut public.

Sans doute :

Sous la pression de la Commune, l'Assemblée autorisa les municipalités à procéder à des « visites domiciliaires »; les prisons se remplirent de suspects.

Mais Isaac écrit de la Commune qu'elle « représentait le peuple vainqueur » et il n'établit aucun lien direct entre l'emprisonnement des suspects et le massacre puisqu'au contraire, " pour les juger, on institua un Tribunal criminel ». Plus encore, l'annonce de la trahison de La Fayette et de l'investissement de Verdun, si elle suscite une forte émotion, se traduit d'abord par une mobilisation accrue : la Commune « invita les Parisiens à former une armée de 600000 hommes ». C'est à cette fin qu'est rapporté le célèbre discours de Danton ponctué par la formule «de l'audace, encore de l'audace, toujours de l'audace», alors qu'Aimond le plaçait en annonce des massacres, le redoublant des menaces proférées par Marat à l'encontre des ennemis de la Patrie, me-

14. «En face de l'Assemblée impuissante et réduite à moins de 300 députés, la nouvelle Commune de Paris (288 membres) érigea audacieusement son pouvoir usurpé. Sur elle doit peser la principale responsabilité des odieux massacres de Septembre ». 
naces désignant à la vindicte populaire « les malheureux enfermés dans les prisons ». Isaac rompt alors le cours de son récit par un nouveau paragraphe qui s'ouvre sur le constat navré que «cet élan patriotique fut souillé par une abominable tuerie, les massacres dans les prisons ». Plus encore que lors du récit de la mort du gouverneur de la Bastille, l'épisode sanglant est résolument exclu du déroulement normal de l'évènement. Pour la première fois, dans la description de cet épisode, Isaac ne le cède en rien à Aimond dans la rigueur du propos, recourant à un vocabulaire de condamnation indignée inhabituel chez lui. Il s'en prend lui aussi à Marat, qui « fit afficher partout des placards qui conseillaient au peuple de se faire justice lui-même », et constate que " ces conseils sanguinaires furent suivis à la lettre ». Aussi le récit des massacres est-il d'une tonalité très proche de celui de Aimond :

Le massacre commença le 2 septembre dans la cour de la prison de l'Abbaye, où l'on avait amené des prêtres réfractaires. Pendant quatre jours et quatre nuits, jusqu'au 6 septembre, sous la direction du Comité de surveillance, et devant un simulacre de tribunal populaire, des bandes d'égorgeurs, de 150 à 200 individus environ, poursuivirent méthodiquement la hideuse besogne dans toutes les prisons, à l'Abbaye, à la Force, au Châtelet, à la Conciergerie, etc. Ils ne s'arrêtèrent que quand les prisons furent vides ; 1100 personnes environ, des prêtres, des vieillards, des femmes, entre autres une amie de la reine, la princesse de Lamballe, avaient péri.

Ces derniers détails quant aux personnes exécutées (des femmes et des vieillards) relèvent même davantage que chez Aimond (qui ne qualifiait que les prêtres réfractaires, conservant somme toute à l'évènement son caractère politique) la gratuité du massacre. Ce qui explique peut-être la virulence exceptionnelle de son propos : elle vise à disqualifier irrémédiablement les massacres et à les rejeter irrévocablement de la série des évènements proprement révolutionnaires. Ce que laisse supposer une fois de plus l'énoncé réducteur des auteurs des massacres (150 à 200 personnes), impossibles à confondre avec une quelconque fraction du peuple souverain ${ }^{15}$. Marquer aussi vigoureusement la différence de nature entre les journées révolutionnaires et les massacres de septembre, revient à valider les premières dans le champ politique, alors qu'en traitant dans la continuité ces deux séries d'évènements, Aimond vise tout aussi surement à leur nullité comme actes de souveraineté.

15. Position renforcée par le fait que si Isaac évoque l'opposition des Girondins aux « septembriseurs ", c'est après avoir expliqué que Danton intervint en fin de compte pour faire révoquer les mandats d'arrêts lancés par le Comité de surveillance à l'instigation de Marat contre Roland et Brissot (en somme pour protéger les représentants de la nation), et sans expliciter davantage le terme de « septembriseurs ». Alors qu'Aimond souligne plutôt que Danton tenta d'excuser les massacres comme un acte de «justice populaire », et que les Girondins se séparèrent des « Jacobins septembriseurs ». 
Nous retiendrons quatre conditions auxquelles l'émeute révolutionnaire doit répondre pour trouver sa légitimation politique. Les trois premières répondent à la question initiale de l'émeute comme acte de souveraineté. Pour qu'il y ait acte de souveraineté, et donc acceptation politique de l'émeute, il faut qu'elle puisse être interprétée comme une réaction spontanée à l'oppression du corps politique, qu'elle manifeste l'unanimité et l'indivisibilité de ce corps, et qu'elle soit garantie par l'union du peuple souverain et de ses représentants légitimes (qu'il s'agisse de l'Assemblée des représentants, comme au 14 juillet 1789, ou de corps constitués dans la dynamique même de l'émeute révolutionnaire, telle la commune insurrectionnelle). Par ailleurs, elle exclut toute forme de violence en dehors des combats qu'elle implique.

Quelles que soient leurs oppositions, les deux auteurs construisent leurs positions respectives sur les mêmes présupposés, et c'est en cela que nous considérons ces présupposés comme formant une représentation négative de l'émeute révolutionnaire : négative parce qu'elle s'inscrit en creux dans les argumentations développées; négative encore parce qu'elle porte un même refus de l'émeute pour elle-même. Représentation négative enfin, parce qu'elle est partagée par les deux auteurs au delà (ou en deçà ?) de leurs partis pris historiographiques et politiques.

C'est ainsi qu'Aimond souscrit implicitement aux conditions énoncées dans sa condamnation sans restriction de l'émeute révolutionnaire. Il n'y voit que l'action d'un groupe de factieux. Ceux-ci dirigent leurs coups contre les autorités légitimes sans relais institutionnel possible (l'attaque contre le pouvoir royal en 1789 n'est pas «compensée » comme chez Isaac, puisqu'Aimond n'établit aucun lien direct entre la défense de l'Assemblée et l'insurrection parisienne, ni entre celle-ci et la municipalité). Aimond ne met pas en cause le "peuple» mais toujours une fraction de ce peuple, et la plus vile. Il rejette enfin l'émeute au grief d'une violence qu'Isaac s'accorde, par son souci constant à l'exclure du cours normal des évènements, à reconnaitre illégitime.

Ajoutons que la relation paradoxale de l'émeute aux autorités constituées, chez Isaac, conforte cette comparaison entre les présupposés de l'argumentation des auteurs. Tout se passe comme si la possibilité d'une médiation représentative de l'émeute (c'est le sens que nous donnons à l'insistance de l'auteur sur la formation de la municipalité et de la Garde nationale, le 13 juillet 1789), aussi fragile soit-elle, rendait seule sup- 
portable l'intervention directe d'un peuple en dernière instance perturbateur de l'ordre social. La validation de l'émeute étant en définitive entendue comme palliatif du véritable acte de souveraineté légitime (accompli par le biais d'une représentation). C'est le dernier aspect de la représentation négative partagée et mobilisée de manière identique chez les deux auteurs. 